\title{
Longitudinal study of interventional radiology activity in a large metropolitan Italian tertiary care hospital: how the COVID-19 pandemic emergency has changed our activity
}

\author{
Roberto lezzi ${ }^{1,2}$ (D) lacopo Valente ${ }^{1} \cdot$ Alessandro Cina ${ }^{1} \cdot$ Alessandro Posa $^{1} \cdot$ Andrea Contegiacomo $^{1}$. \\ Andrea Alexandre ${ }^{1}$. Francesco $\mathrm{D}^{\prime}$ Argento $^{1}$ • Emilio Lozupone ${ }^{1}$. Michele Barone ${ }^{1} \cdot$ Francesca Giubbolini $^{1}$. \\ Luca Milonia ${ }^{1}$ - Andrea Romi ${ }^{1}$ - Anna Rita Scrofani ${ }^{1}$ - Alessandro Pedicelli ${ }^{1} \cdot$ Riccardo Manfredi $^{1,2} \cdot$ Cesare Colosimo $^{1,2}$
}

Received: 21 May 2020 / Revised: 2 June 2020 / Accepted: 17 June 2020 / Published online: 30 June 2020

(C) European Society of Radiology 2020

\begin{abstract}
Objectives To retrospectively analyze interventional radiology (IR) activity changes in the COVID-19 era and to describe how to safely and effectively reorganize IR activity.

Methods All IR procedures performed between January 30 and April 8, 2020 (COVID-era group) and the same 2019 period (non-COVID-era group) were retrospectively included and compared. A sub-analysis for the lockdown period (LDP: $11 \mathrm{March}-$ 8 April) was also conducted. Demographic, hospitalization, clinical, and procedural data were obtained for both groups and statistically compared with univariable analysis.

Results A total of 1496 procedures (non-COVID era, 825; COVID era, 671) performed in 1226 patients (64.9 \pm 15.1 years, 618 women) were included. The number of procedures decreased by $18.6 \%$ between 2019 and 2020 (825 vs $671, p<.001)$, with a reduction by $48.2 \%$ in LDP ( 188 vs $363, p<.0001)$. In the LDP COVID era, bedside procedures were preferred $(p=.013)$, with an increase in procedures from the intensive care unit compared with the emergency department and outpatients $(p=.048)$, and an increased activity for oncological patients $(p=.003)$. No incidents of cross-infection of non-infected from infected patients and no evidence of COVID-19 infection of healthcare workers in the IR service was registered.

Conclusions Coronavirus disease outbreak changed the interventional radiology activity with an overall reduction in the number of procedures. However, this study confirms that interventional radiology continuum of care can be safely performed also during the pandemic, following defined measures and protocols, taking care of all patients.

Key Points

- Coronavirus disease pandemic determined a reduction of interventional radiology activity as compared to the same period of the previous year.

- Interventional radiology procedures for life-threatening conditions and non-deferrable oncologic treatments were prioritized as opposed to elective procedures.

- Strict adoption of safe procedures allowed us to have until now no incidents of cross-infection of non-infected from infected patients and no evidence of COVID-19 infection of HCWs in the IR service.
\end{abstract}

Keywords Interventional radiology $\cdot$ Neoplasms $\cdot$ Virus diseases $\cdot$ Infections $\cdot$ Safety

Roberto Iezzi

roberto.iezzi.md@gmail.com; roberto.iezzi@policlinicogemelli.it

1 Fondazione Policlinico Universitario “A. Gemelli” - IRCCS, Dipartimento di Diagnostica per Immagini, Radioterapia, Oncologia ed Ematologia - Area di Diagnostica per Immagini, UOC Radiologia Diagnostica e Interventistica Generale, L.go A. Gemelli 8, 00168 Rome, Italy

2 Università Cattolica del Sacro Cuore, L.go F. Vito 1, 00168 Rome, Italy

\section{Abbreviations}

COVID-19 Coronavirus disease

HCWs Healthcare workers

IR Interventional radiology

LDP Lockdown period 


\section{Introduction}

The World Health Organization declared the 2019-2020 coronavirus disease (COVID-19) outbreak a Public Health Emergency of International Concern on 30 January 2020 and a pandemic on 11 March 2020 [1, 2]. In Italy, the first cluster of SARS-CoV-2-positive patients was reported in Lombardy, a region of northern Italy, on 21 February, and after entering, COVID-19 has been spreading fast [3-7]. The outbreak has caused the Italian government to take severe protective measures: on 9 March, a national quarantine was imposed, restricting population movement, whereas on 21 March, a further enlargement of the "lockdown" was announced, shutting down all unnecessary businesses and industries [8-10].

In this scenario, delivering healthcare is extremely challenging given the competing risks of death from acute/subacute diseases, such as cancer or cardiovascular diseases, with death from COVID-19 infection [11]. To ensure both healthcare worker (HCW) and patient safety, long-term sustainability of services and resource availability were shifted to patients critically ill with COVID-19, delaying non-emergency procedures $[4,12]$. However, efforts should be made not to compromise the prognosis of patients with high-risk acute/subacute diseases, still requiring a high standard of care. Furthermore, hospital organizations need to protect healthcare personnel from the risk of infection and limit the possibility for HCWs to transmit the disease [13].

Interventional radiology (IR) has a critical role in the management of patients within the healthcare system, being involved in the treatment of both urgent and chronic diseases and having a pivotal role within the therapeutic pathway of many oncologic patients, also during a pandemic [14]. On the other hand, the risk of transmission is related to the degree of contact between patients and HCWs. Therefore, certain subspecialties like IR entail a greater risk of acquiring and transmitting infection due to the close patient contact and invasive patient care these services provide [15-17].

This makes it imperative to develop and set up guidelines in order to limit transmission, while optimizing resources, rearranging work site and health force, and enforcing segregation and disinfection parameters [18].

The primary aims of our study were to perform a descriptive analysis and longitudinal study of IR activity performed in our metropolitan tertiary care hospital during the COVID19 pandemic, and compare the number and characteristics of IR procedures we carried out with those performed in the same period of 2019. The secondary objective was to describe how to reorganize IR activity to effectively deal with the COVID-19 spread.

\section{Materials and methods}

\section{Study design and setting}

This is a retrospective observational study conducted in a large tertiary-care 1526-bed teaching hospital in Rome (Italy). Our center is a hub for emergency medicine, adult/ pediatric traumas, and oncological and cardiovascular diseases, as well as neurovascular and body interventional radiology. Our center was also designated as a hub even for COVID-19 infection treatment.

All consecutive patients admitted to our department to undergo an interventional radiology procedure between January 30 and April 8, 2020, were included (COVID-era group). This IR activity was compared with that performed in the same time range of the previous year (2019; non-COVID-era group).

For each included patient, demographic and clinical characteristics as well as procedural data were retrospectively recorded by reviewing the patient's charts and electronic medical records and analyzed. Demographic, clinical, and procedural variables are shown in Table 1.

For the COVID-era group, COVID-19 status (negative/low risk, suspected/intermediate-to-high risk, positive) was also registered before the procedure and during the admission (in case of a late positive result), based on clinical/laboratory data and SARS-CoV-2 culture test (Table 1).

A further analysis was also performed considering two subgroups of patients, based on a temporal criterion (before and after 11 March): the so-called pre-lockdown period subgroup (pre-LDP - 30 January-10 March) and lockdown period subgroup (LDP-11 March-8 April, 2020); these subgroups of patients were also compared with those in a similar period of 2019.

This study followed the protocol and the principles of the Declaration of Helsinki, in accordance with the International Conference on Harmonization Tripartite Guideline for Good Clinical Practice, and was approved by our local Institutional Review Board. Written informed consent was obtained from all subjects prior to any treatment.

\section{IR activity reorganization to reduce virus spread}

To comply with government directives, on 11 March 2020, our department has implemented organizational changes to modulate the elective IR activity. One angiography suite has been dedicated to COVID-19 patients whereas the other has been dedicated to elective IR for about $6 \mathrm{~h}$ a day with an oncall activity for emergencies in the remaining $18 \mathrm{~h}$ of each day. Only one weekly slot for CT-guided activity has been planned whereas dedicated US-guided slots, usually performed in ultrasound rooms, were canceled. In detail, during LDP, USguided procedures were performed bedside, to reduce 
Table 1 Patients' demographic, clinical, and procedural data

\begin{tabular}{ll}
\hline Clinical and demographic data & Procedural data \\
\hline Sex & Type of procedure (diagnostic or therapeutic) \\
Age & Urgency of procedure (elective or emergency) \\
Date and time of admission & Guidance (fluoroscopy, US, CT) \\
Symptoms at the time of procedure & Oncological setting (procedures in non-oncologic patients-for \\
(fever, respiratory symptoms) & benign diseases, procedures in oncologic patients—not fo- \\
& cused on the tumor, oncological treatments-procedures fo- \\
& cused on the tumor) \\
Symptoms during the last 15 days & Treated body part \\
(fever, respiratory symptoms) & (head, thorax, abdomen, bone, other) \\
Past medical history & Ward of provenance (emergency department, intensive care \\
(previous or ongoing malignancy) & unit, general wards) \\
Epidemiologic link & Delay between last diagnostic imaging and IR procedure (for \\
(possible contact with COVID-19 subjects or & emergency procedures) \\
coming from high-risk areas) & Number of bedside US-guided procedures \\
Patient risk stratification* & \\
\hline
\end{tabular}

*Patients were stratified into low risk/negative (no symptoms or epidemiologic link), intermediate risk/suspect (presence of symptoms at the time of procedure or during the previous 15 days, or epidemiologic link), high risk/ suspect (presence of symptoms and epidemiologic link), and positive (positive test for SARS-CoV-2) contamination, or in the angiography suite whenever possible, to reduce contamination and the number of IR consultants in the hospital. Based on these organizational changes, one IR consultant was actively dedicated to IR angiographic or CT slots in the morning with another one on-call for emergency in the remaining hours of the day. Access to residents in the angio-suite has been reduced as much as possible and video consultations were offered to patients and clinicians. Furthermore, access to our department has been restricted to visitors, students, external colleagues, and specialists.

To reduce virus diffusion through infected patients, each patient has been equipped with a surgical mask, regardless of risk factors. Patients who would benefit from a bedside procedure have been treated in their ward in order to limit their movement. Any effort has been put in place to reduce waiting time in preoperative holding areas.

All patients admitted to our hospital to undergo IR elective procedures always performed accurate triage, chest X-ray, and a rhino-pharyngeal swab, regardless of symptoms and risk factors, to avoid room contamination and unprotected HCWs exposure; testing all patients allowed us to create three different dedicated routes, for negative, doubt/unknown waiting for results, and positive patients.

To avoid useless waste, the use of personal protective equipment was optimized, based on patient risk, as previously defined, or procedure type: standard precautions (surgical cap, surgical mask, goggles, work uniform, and latex gloves) for low-risk patients, airborne precautions (disposable surgical cap, medical protective mask FFP2/N95, work uniform, disposable latex gloves, water-resistant disposable medical protective uniform, and full-face protective device) for intermediate- or high-risk patients, as well as for COVID- positive patients, since the need of endotracheal intubation could be surely excluded, for potential intraprocedural complication. It is mandatory to highlight that there are no IR aerosol-generating procedures (AGP), but for procedures like lung biopsy, pleural drainage, lung ablation, or bronchial artery embolization, patients could experience hemoptysis during the procedures which can incite violent coughing and aerosolization; hence, it may be considered an AGP.

\section{"Safety" healthcare worker analysis}

Test was only performed in symptomatic HCWs or after unprotected exposure to a patient with confirmed or suspected COVID-19 regardless of symptoms [19]; in detail, no widespread testing was used in our hospital for HCWs.

The number of IR team components (composed of 9 IRs, 9 technicians, 10 nurses, and 2 administrative personnel) who experienced symptoms suspected for COVID-19 or underwent COVID-19 test, or underwent preventive quarantine, or was COVID-19 positive or hospitalized for COVID19 was registered.

Given that the presumed potential time lag between exposure to coronavirus and clinical infection is up to about 14 days, data were recorded till April 22 in order not to miss late healthcare worker or patient infections derived from exposures up to April 8.

\section{Statistical analysis}

Demographic, clinical, and procedural characteristics of all patients for both the non-COVID era and the COVID era were collected. A univariable analysis was conducted to compare 
demographics, clinical, and procedural characteristics of the two periods. For continuous variables, means, medians, interquartile ranges (IQR), and standard deviations (SD) are presented and $p$ values calculated with $t$ test or Wilcoxon ranksum depending on normality distribution (tested with Shapiro Wilk's). For categorical measures, frequencies and percentages are presented and $p$ values calculated with Fisher's exact test or $\chi^{2}$ as appropriate. No adjustments were made for multiple comparisons. Statistical significance threshold was set at $5 \%$. The statistical analysis has been performed using STATA 15.1 (StataCorp LLC).

\section{Results}

A total of 1496 procedures, performed on a total of 1226 patients, were analyzed, 825 in the non-COVID era and 671 in the COVID era. Patients' clinical, radiological, and procedural characteristics are shown in Table 2.

An overall $18.6 \%$ decreasing of the procedures between 2019 and 2020 was observed. Demographics, clinical, and procedural characteristics were comparable for the two groups except for ward of provenance: emergency department (13.9\% vs $9.8 \%)$, intensive care units $(1.3 \%$ vs $2.5 \%)$, and outpatients $(6.3 \%$ vs $8.8 \%)(p=.01)$. US, CT, and US + fluoroscopic guidance were more frequently used in 2019, while fluoroscopic guidance was more frequently used in 2020 $(p=.03)$, with head, abdomen, and bone procedures being more frequent in $2020(p=.01)$.

In both 2019 and 2020, most of the procedures were therapeutic $(84.5 \%$ and $82.1 \%)$ with a similar number of procedures performed in the emergency care setting $(33.3 \%$ and $30.1 \%$ ).

Furthermore, when considering emergency procedures, there was no significant difference in the delay between imaging and intervention between 2019 and 2020 (90 vs 95 min, $p=0.7)$.

No differences were found within the oncological settings category, for procedures performed in non-oncologic patients (benign diseases), procedures performed in oncologic patients but not focused to the tumor (such as drainage, nephrostomy, caval filters), or oncological procedures focused on the tumor (such as ablation, chemo/radioembolization).

\section{Subgroup analysis: pre-lockdown period (pre-LDP)}

In the pre-LDP (30 Jan-10 Mar), a total of 945 procedures were analyzed: 462 in 2019 and 483 in 2020 with a relative increase of $4.5 \%$. Patients' clinical, radiological, and procedural characteristics are shown in Table 3. Demographics and procedural characteristics were comparable, except for ward of provenance: emergency departments (13\% vs 9.7\%), general ward $(78.6 \%$ vs $76.6 \%)$, outpatients $(6.5 \%$ vs $11 \%)$, and intensive care units $(1.95 \%$ vs $2.69 \%)(p=.040)$. CT and US + fluoroscopic guidance were more frequently used in 2019 while US, US + CT, and fluoroscopic guidance were more frequently used in 2020 ( $p=.006)$. Head, abdomen and bone procedures were more frequent in $2020(p=.013)$, with no bedside procedures performed during 2020 (vs 12/483, $2.6 \% ; p<.001)$.

\section{Subgroup analysis: lockdown period (pre-LDP)}

When considering the lockdown period (11 Mar-8 Apr), a total of 551 procedures were analyzed: 363 in 2019 and 188 in 2020 with a $48.2 \%$ relative decrease. Patients' clinical, radiological, and procedural characteristics are shown in Table 4 and Table 5. Demographics and procedural characteristics were comparable, except for ward of provenance: emergency departments $(15.15 \%$ vs $10.11 \%)$, intensive care units $(0.55 \%$ vs $2.13 \%)$, and outpatients ( $6.06 \%$ vs $3.19 \%)(p=.048)$; also, oncological setting differed between the two periods, with a decreasing of non-oncologic patients (43.25\% vs $28.19 \%$ ) and an increasing of both procedures in oncologic patients $(38.02 \%$ vs $48.94 \%)$ and oncologic treatments $(18.73 \%$ vs $22.87 \%)(p=.003)$. Bedside procedures were more common during 2020 than 2019 (2.13\% vs $0 \% ; p=.01)$.

\section{Suspected or COVID-19-positive patients}

All patients referring to our emergency department underwent nasopharyngeal swab for SARS-CoV-2 culture test; all patients, regardless of the risk class, were equipped with a surgical mask.

During the whole COVID era, nine patients $(9 / 671,1.34 \%)$ were classified as suspected while one patient $(1 / 671,0.15 \%)$ was COVID-19 positive; considering the sole lockdown period, we had 7 suspected patients $(7 / 188,3.72 \%)$ and one COVID-19-positive patient (1/188, 0.53\%).

\section{"Safety" healthcare workers analysis}

COVID-19 testing was performed only in 2 nurses and 2 technicians $(4 / 30,13.3 \%)$; all of them were asymptomatic so no one experienced a preventive quarantine. Test was negative in all HCWs. No HCWs of our team was hospitalized for COVID-19.

\section{Discussion}

The coronavirus disease worldwide outbreak has led to a dramatic challenge for all healthcare systems [20]. IR services would be prepared to adequately perform elective or emergency procedures also under these extreme circumstances. The main issue is related to the protection of patients and 
Table 2 Baseline demographics, clinical, and procedural characteristics

\begin{tabular}{|c|c|c|c|c|}
\hline Variables & Overall & Non-COVID era (2019) & COVID era (2020) & $p$ value \\
\hline Patients & 1226 & $676(55.1 \%)$ & $550(44.9 \%)$ & \\
\hline Age (years) & $64.93 \pm 15.14$ & $64.87 \pm 15.7$ & $65.00 \pm 14.39$ & .87 \\
\hline Women & $618(50.4 \%)$ & $340(50.3 \%)$ & $278(50.5 \%)$ & .89 \\
\hline Number of Procedures & 1496 & $825(55.15 \%)$ & $671(45.85 \%)$ & $<.001$ \\
\hline Ward of provenance (per procedure) & & & & .01 \\
\hline Emergency department & $181(12.10 \%)$ & $115(13.94 \%)$ & $66(9.84 \%)$ & \\
\hline General ward & $1176(78.61 \%)$ & $647(78.42 \%)$ & $529(78.84 \%)$ & \\
\hline Outpatients & $111(7.42 \%)$ & $52(6.30 \%)$ & $59(8.79 \%)$ & \\
\hline Intensive care units & $28(1.87 \%)$ & $11(1.33 \%)$ & $17(2.53 \%)$ & \\
\hline Procedural guidance & & & & .03 \\
\hline Fluoroscopy & $703(46.99 \%)$ & $367(44.48 \%)$ & $336(50.07 \%)$ & \\
\hline US & $251(16.78 \%)$ & $141(17.09 \%)$ & $110(16.39 \%)$ & \\
\hline $\mathrm{CT}$ & $136(9.09 \%)$ & $87(10.55 \%)$ & $49(7.30 \%)$ & \\
\hline $\mathrm{US}+\mathrm{CT}$ & $8(0.53 \%)$ & $2(.24 \%)$ & $6(.89 \%)$ & \\
\hline US + fluoroscopy & $398(26.60 \%)$ & $228(27.64 \%)$ & $170(25.34 \%)$ & \\
\hline Bedside procedures & $16(1.07 \%)$ & $12(1.45 \%)$ & $4(.60 \%)$ & .13 \\
\hline Type of procedure & & & & .22 \\
\hline Diagnostic & $248(18.1 \%)$ & $128(15.5 \%)$ & $120(17.9 \%)$ & \\
\hline Therapeutic & $1248(83.42 \%)$ & $697(84.48 \%)$ & $551(82.12 \%)$ & \\
\hline Body part & & & & .01 \\
\hline Head & $132(8.82 \%)$ & $65(7.88 \%)$ & $67(9.99 \%)$ & \\
\hline Chest & $123(8.22 \%)$ & $81(9.82 \%)$ & $42(6.26 \%)$ & \\
\hline Abdomen & $1007(67.31 \%)$ & $549(66.55 \%)$ & $458(68.26 \%)$ & \\
\hline Bone & $62(4.14 \%)$ & $27(3.27 \%)$ & $35(5.22 \%)$ & \\
\hline Others & $172(11.50 \%)$ & $103(12.48 \%)$ & $69(10.28 \%)$ & \\
\hline Urgency of procedure & & & & .18 \\
\hline Elective & $1019(68.1 \%)$ & $550(66.7 \%)$ & $469(69.9 \%)$ & \\
\hline Emergency & $477(31.9 \%)$ & $275(33.3 \%)$ & $202(30.1 \%)$ & \\
\hline Delay time-dependent procedures (min) & $90(40-180)$ & $90(40-330)$ & $95(52.5-130.5)$ & .70 \\
\hline Oncological setting & & & & .35 \\
\hline Procedures in non-oncologic patients & $586(39.17 \%)$ & $334(40.48 \%)$ & $248(36.96 \%)$ & \\
\hline Procedures in oncologic patients & $642(42.91 \%)$ & $342(41.45 \%)$ & $300(44.71 \%)$ & \\
\hline Oncologic treatments & $268(17.91 \%)$ & $149(18.06 \%)$ & $123(18.33 \%)$ & \\
\hline
\end{tabular}

CT computed tomography, min minutes, US ultrasound

healthcare workers (HCW) from being contaminated or infected, while providing safe IR care for patients $[15,18]$. With this attempt, many papers were focused on sharing measures and potential challenges that are most relevant to the practice of IR $[15,17,21]$. However, to date, there are no published data regarding the real impact of COVID-19 pandemic emergency on IR activity, in particular showing if measures and challenges applied were able to obtain clinical results in terms of safety for both patients and HCW.

Based on this attempt, our retrospective study was performed analyzing our activity from January 30 to April 8, 2020 (COVID era), compared with the same activity obtained in 2019 (non-COVID era). We also compared two subgroups of patients treated before or after March 11, when a lockdown, provided by government after World Health Organization's pandemic declaration, was imposed. It became mandatory to postpone outpatient activity and elective procedures, in particular for benign diseases, especially in older patients and those with comorbidities [22].

However, all procedures with a survival benefit needed to be maintained and prioritized whenever possible. For all these procedures that could not be postponed, accurate selection/ stratification of patients is mandatory to avoid unprotected accidental contamination of angio-suite and healthcare workers.

Our data confirmed that IR activity was significantly reduced in the overall COVID era by $20 \%$. However, reduction 
Table 3 Baseline demographics and clinical and procedural characteristics for the procedure performed before the "lockdown period" (pre-LDP: Jan 30-Mar 10)

\begin{tabular}{|c|c|c|c|c|}
\hline Variables & Overall & Non-COVID era LDP & COVID era LDP & $p$ value \\
\hline Patients & 725 & $346(47.73 \%)$ & $379(52.27 \%)$ & \\
\hline Age (years) & $64.93 \pm 15.14$ & $64.98 \pm 15.82$ & $65.04 \pm 14.44$ & .516 \\
\hline Women & $474(50.16 \%)$ & $225(48.70 \%)$ & $249(51.55 \%)$ & .381 \\
\hline Number of procedures & 945 & $462(48.89 \%)$ & $483(51.11 \%)$ & \\
\hline Ward of provenance (per procedure) & & & & .040 \\
\hline Emergency department & $107(11.32 \%)$ & $60(12.99 \%)$ & $47(9.73 \%)$ & \\
\hline General ward & $733(77.57 \%)$ & $363(78.57 \%)$ & $370(76.60 \%)$ & \\
\hline Outpatients & $83(8.78 \%)$ & $30(6.49 \%)$ & $53(10.97 \%)$ & \\
\hline Intensive care units & $22(2.33 \%)$ & $9(1.95 \%)$ & $13(2.69 \%)$ & \\
\hline Procedural guidance & & & & .006 \\
\hline Fluoroscopy & $447(47.30 \%)$ & $200(43.29 \%)$ & $247(51.14 \%)$ & \\
\hline US & $152(16.08 \%)$ & $70(15.15 \%)$ & $82(16.98 \%)$ & \\
\hline $\mathrm{CT}$ & $96(10.16 \%)$ & $60(12.99 \%)$ & $36(7.45 \%)$ & \\
\hline $\mathrm{US}+\mathrm{CT}$ & $5(0.53 \%)$ & $1(0.22 \%)$ & $4(0.83 \%)$ & \\
\hline US + fluoroscopy & $245(25.93 \%)$ & $131(28.35 \%)$ & $114(23.60 \%)$ & \\
\hline Bedside procedures & $12(1.27 \%)$ & $12(2.60 \%)$ & 0 & $<.001$ \\
\hline Type of procedure & & & & .168 \\
\hline Diagnostic & $153(16.19 \%)$ & $67(14.50 \%)$ & $86(17.81 \%)$ & \\
\hline Therapeutic & $792(83.81 \%)$ & $395(85.50 \%)$ & $397(82.19 \%)$ & \\
\hline Body part & & & & .013 \\
\hline Head & $86(9.10 \%)$ & $34(7.36 \%)$ & $52(10.77 \%)$ & \\
\hline Thorax & $78(8.25 \%)$ & $50(10.82 \%)$ & $28(5.80 \%)$ & \\
\hline Abdomen & $620(65.61 \%)$ & $301(65.15 \%)$ & $319(66.05 \%)$ & \\
\hline Bone & $49(5.19 \%)$ & $19(4.11 \%)$ & $30(6.21 \%)$ & \\
\hline Others & $112(11.85 \%)$ & $58(12.55 \%)$ & $54(11.18 \%)$ & \\
\hline Emergency procedures & & & & .661 \\
\hline Elective & $669(70.79 \%)$ & $324(70.13 \%)$ & $345(71.43 \%)$ & \\
\hline Emergency & $276(29.21 \%)$ & $138(29.87 \%)$ & $138(28.57 \%)$ & \\
\hline Delay time-dependent procedures (min) & $226(70-463)$ & $246(68-540)$ & $179(80-360)$ & .291 \\
\hline Oncologic classes & & & & .799 \\
\hline Procedures in non-oncologic patients & $372(39.37 \%)$ & $177(38.31 \%)$ & $195(40.37 \%)$ & \\
\hline Procedures in oncologic patients & $412(43.60 \%)$ & $204(44.16 \%)$ & $208(43.06 \%)$ & \\
\hline Oncologic treatments & $161(17.04 \%)$ & $81(17.53 \%)$ & $80(16.56 \%)$ & \\
\hline
\end{tabular}

$C T$ computed tomography, LDP lockdown period, min minutes, US ultrasound

was obtained only in the lockdown period, with 188 procedures performed in 4 weeks (versus 363 for non-COVID era) and more than $48 \%$ reduction of activity registered. These results highlighted that variations in procedure numbers were only due to the lockdown secondary to COVID emergency, and not to year-on-year variability, as demonstrated by the relative slight increase $(4.5 \%)$ obtained in the pre-LDP. In detail, data collected in pre-LDP showed similar trend in terms of oncological classes (procedures performed in nononcologic or oncologic patients).

Our activity was also almost homogeneous when considering the overall period; in detail, demographics and procedural characteristics were almost comparable for the two groups in terms of type (therapeutic for more than $80 \%$ of them), rate of emergency ( $33.3 \%$ vs $30.1 \%$ ), indication, and oncological setting. Interesting results were obtained when considering the LDP only, in which we obtained significant differences for oncological setting, with about $72 \%$ of procedures performed in oncologic patients (versus $57 \%$ ), prioritizing procedure for life-threatening conditions, non-deferrable oncologic treatments, and organsaving procedures, with the same mean number of elective procedures for session in oncologic patients when compared to the same period in 2019 . 
Table 4 Baseline demographics and clinical and procedural characteristics for the procedure performed during the "lockdown period"

\begin{tabular}{|c|c|c|c|c|}
\hline Variables & Overall & Non-COVID era LDP & COVID era LDP & $p$ value \\
\hline Patients & 501 & $330(65.87 \%)$ & $171(34.13 \%)$ & \\
\hline Age (years) & $64.88 \pm 14.30$ & $64.73 \pm 15.64$ & $64.88 \pm 14.30$ & .91 \\
\hline Women & $281(51.00 \%)$ & $190(52.34 \%)$ & $91(48.40 \%)$ & .38 \\
\hline Number of procedures & 551 & $363(65.88 \%)$ & $188(34.12 \%)$ & $<.001$ \\
\hline Ward of provenance (per procedure) & & & & .048 \\
\hline Emergency department & $74(13.43 \%)$ & $55(15.15 \%)$ & $19(10.11 \%)$ & \\
\hline General ward & $443(80.40 \%)$ & $284(78.24 \%)$ & $159(84.57 \%)$ & \\
\hline Outpatients & $28(5.08 \%)$ & $22(6.06 \%)$ & $6(3.19 \%)$ & \\
\hline Intensive care units & $6(1.09 \%)$ & $2(0.55 \%)$ & $4(2.13 \%)$ & \\
\hline Procedural guidance & & & & .49 \\
\hline Fluoroscopy & $256(46.46 \%)$ & $167(46.01 \%)$ & $89(47.34 \%)$ & \\
\hline US & $99(17.97 \%)$ & $71(19.56 \%)$ & $28(14.89 \%)$ & \\
\hline $\mathrm{CT}$ & $40(7.26 \%)$ & $27(7.44 \%)$ & $13(6.91 \%)$ & \\
\hline $\mathrm{US}+\mathrm{CT}$ & $3(0.54 \%)$ & $1(0.28 \%)$ & $2(1.06 \%)$ & \\
\hline US + fluoroscopy & $153(27.77 \%)$ & $97(26.72 \%)$ & $56(29.79 \%)$ & \\
\hline Bedside procedures & $4(.73 \%)$ & 0 & $4(2.13 \%)$ & .01 \\
\hline Type of procedure & & & & .706 \\
\hline Diagnostic & $95(17.24 \%)$ & $61(16.80 \%)$ & $34(18.09 \%$ & \\
\hline Therapeutic & $456(82.76 \%)$ & $302(83.20 \%)$ & $154(81.91 \%)$ & \\
\hline Body part & & & & .54 \\
\hline Head & $46(8.35 \%)$ & $31(8.54 \%)$ & $15(7.98 \%)$ & \\
\hline Thorax & $45(8.17 \%)$ & $31(8.54 \%)$ & $14(7.45 \%)$ & \\
\hline Abdomen & $387(70.24 \%)$ & $248(68.32 \%)$ & $139(73.94 \%)$ & \\
\hline Bone & $13(2.36 \%)$ & $8(2.20 \%)$ & $5(2.66 \%)$ & \\
\hline Others & $60(10.89 \%)$ & $45(12.40 \%)$ & $15(7.98 \%)$ & \\
\hline Emergency procedures & & & & .39 \\
\hline Elective & $350(63.52 \%)$ & $226(62.26 \%)$ & $124(65.96 \%)$ & \\
\hline Emergency & $201(36.48 \%)$ & $137(37.74 \%)$ & $64(34.04 \%)$ & \\
\hline Delay time-dependent procedures (min) & $86.5(40-180)$ & $50(40-180)$ & $100(55-180)$ & \\
\hline Oncologic classes & & & & .003 \\
\hline Procedures in non-oncologic patients & $210(38.11 \%)$ & $157(43.25 \%)$ & $53(28.19 \%)$ & \\
\hline Procedures in oncologic patients & $230(41.74 \%)$ & $138(38.02 \%)$ & $92(48.94 \%)$ & \\
\hline Oncologic treatments & $111(20.15 \%)$ & $68(18.73 \%)$ & $43(22.87 \%)$ & \\
\hline
\end{tabular}

$C T$ computed tomography, LDP lockdown period, min minutes, US ultrasound

Unfortunately, the effects of COVID-19 are not solely limited to the treatment of patients with cancer, but will also hit the entire healthcare community, with consequences for prevention/screening programs and early diagnosis of diseases, management of cardiovascular pathologies, scheduled operations, appointments/consultations that were postponed to prioritize hospital beds and care for COVID-19 pandemic. COVID also had inevitable consequences for research, education, and collaboration. Furthermore, COVID-19 also deeply influenced patients' perception of medical priorities and risk of contagion: patients could be afraid of coming to the hospital and some of them could have decided to stay at home even if their conditions deserved urgent evaluation. The long-term consequences of this scenario are difficult to estimate, as a long follow-up duration will be required to reveal the effects of delayed diagnosis and management/treatment of diseases.

It is interesting to highlight that even if our Hospital was highly exposed to COVID patients, among all treated patients during COVID-period, only $1.5 \%$ of them were suspected or COVID-positive. This data confirmed that COVID-positive patients do not require an excessive burden of IR procedures, highlighting the aim of preserving a regular activity for nonCOVID patients being able to protect both patients and healthcare workers. For this attempt, it is mandatory to follow procedural approaches for the IR workflow, creating different dedicated routes and facilities to reduce contamination, 
Table 5 Types of procedures performed by period of time

\begin{tabular}{|c|c|c|c|c|}
\hline Procedure types & $\begin{array}{l}\text { Non- } \\
\text { COVID } \\
\text { era }\end{array}$ & $\begin{array}{l}\text { COVID } \\
\text { era }\end{array}$ & $\begin{array}{l}\text { Non- } \\
\text { COVID } \\
\text { LDP }\end{array}$ & $\begin{array}{l}\text { COVID } \\
\text { LDP }\end{array}$ \\
\hline Hepatic oncological treatments (ablation/TACE/TARE) & 58 & 49 & 23 & 16 \\
\hline Other ablations & 5 & 2 & 3 & 0 \\
\hline Hepatic/pulmonary biopsy & 59 & 44 & 25 & 15 \\
\hline Other biopsy & 31 & 31 & 18 & 13 \\
\hline Traumatic/post-surgical embolization (bleeding) & 33 & 33 & 16 & 10 \\
\hline $\begin{array}{l}\text { Embolization for benign diseases } \\
\text { (UFE/varicocele/uterine fibroid embol/bronchial) }\end{array}$ & 26 & 5 & 13 & 1 \\
\hline Presurgical embolization (PVE/tumoral embolization) & 4 & 6 & 3 & 2 \\
\hline $\begin{array}{l}\text { Urological therapeutic procedures (nephrostomy/ureteral } \\
\text { stenting) }\end{array}$ & 192 & 145 & 85 & 47 \\
\hline Biliary therapeutic procedures (drainage/stent) & 70 & 64 & 25 & 18 \\
\hline Cholecystostomy & 16 & 10 & 8 & 3 \\
\hline Abdominal drainage & 130 & 104 & 55 & 28 \\
\hline Thoracic drainage & 9 & 5 & 3 & 1 \\
\hline Extracranial/peripheral AVM/DAVF embolization & 8 & 6 & 4 & 0 \\
\hline Abdominal/peripheral revascularization & 88 & 53 & 39 & 9 \\
\hline TIPS & 1 & 5 & 0 & 2 \\
\hline Caval procedures (filter/foreign body removal) & 12 & 13 & 5 & 4 \\
\hline Ischemic stroke & 18 & 29 & 9 & 12 \\
\hline Cerebral aneurysm embolization & 24 & 16 & 11 & 0 \\
\hline Spinal/vertebral procedures & 25 & 34 & 8 & 4 \\
\hline Diagnostic cerebral angiography & 15 & 17 & 10 & 3 \\
\hline
\end{tabular}

$A V M$ artero-venous malformation, $D A V F$ dural artero-venous fistulas, $L D P$ "lockdown period" (between 11 March and 8 April), PVE portal vein embolization, TACE transcatheter arterial chemoembolization, TARE trans-arterial radioembolization, TIPS transjugular intrahepatic portosystemic shunt, UFE uterine fibroid embolization

performing when possible bedside US-guided procedures to avoid moving and transporting suspected/positive patients out of their room/area [23], optimizing the use of appropriate personal protective equipment [24-26].

Strict adoption of safe procedures allowed us to have until now no incidents of cross-infection of non-infected from infected patients and no evidence of COVID-19 infection of HCWs in the IR service up to now [27]. It needs to be highlighted that a main role for obtaining these results were played by rhino-pharyngeal swabs performed to all patients admitted to our hospital to undergo IR elective procedures. Testing patients for COVID allowed us to avoid incidental room contamination and unprotected $\mathrm{HCW}$ exposure, creating three different dedicated routes for negative, doubt/unknown waiting for results, and positive patients.

The main limitation of our study is represented by its retrospective and monocentric nature. Being monocentric, it is strictly center-specific, based on our internal policy, and may not accurately represent national or international health statistics, being not easily repeatable in other centers. However, our aim was not to suggest guidelines or measures to be applied everywhere, but only to perform a descriptive analysis and longitudinal study of IR activity in a tertiary care hospital during the COVID-19 pandemic. To the best of our knowledge, our study is the first work focused on analyzing real impact of COVID-related changes on IR service activity. It is mandatory to underline that, in our experience, reduction of activity was most pronounced in the non-oncologic patient group: the long-term effects of this on the population remain to be seen, and may be the subject of future study.

It is well known that there is no universal solution to healthcare during this crisis. However, IR services face the need to preserve the care for patients, as the duration of this pandemic is hard to predict [28]. In this scenario, protection of patients and physicians is mandatory in order to keep providing the best service in a safe way, in particular when dealing with oncologic patients, which are frail and at high risk for infections.

In conclusion, our study confirmed the high impact of coronavirus disease pandemic on the interventional radiology activity performed in our metropolitan tertiary-care hospital, with a significant reduction of IR procedures due to organizational changes implemented to comply with government directives. However, our experience confirms that the 
interventional radiology continuum of care is needed and can be safely performed also during the pandemic emergency, following defined measures and protocols, taking care of all patients with disease, mainly represented, not just those affected by coronavirus disease.

\section{Compliance with ethical standards}

Guarantor The scientific guarantor of this publication is Roberto Iezzi.

Conflict of interest The authors of this manuscript declare no relationships with any companies, whose products or services may be related to the subject matter of the article.

Statistics and biometry One of the authors has significant statistical expertise (Iacopo Valente).

Informed consent Only if the study is on human subjects:

Written informed consent was obtained from all subjects (patients) in this study.

Written informed consent was waived by the Institutional Review Board.

Ethical approval Institutional Review Board approval was obtained.

\section{Methodology}

- retroprospective

- observational study

- single-center study

\section{References}

1. World Health Organization (2020) WHO statement on the Second Meeting of the International Health Regulations (2005) emergency committee regarding the outbreak of novel coronavirus (2019nCoV). World Health Organization, Geneva.. Available via https://www.who.int/news-room/detail/30-01-2020-statement-onthe-second-meeting-of-the-international-health-regulations-(2005)emergency-committee-regarding-the-outbreak-of-novelcoronavirus-(2019-ncov). Accessed 10 Apr 2020

2. World Health Organization (2020) WHO Director-General's opening remarks at the media briefing on COVID-19 - 11 March 2020. World Health Organization, Geneva. Available via https://www. who.int/dg/speeches/detail/who-director-general-s-openingremarks-at-the-media-briefing-on-covid-19\%2D\%2D-11-march2020. Accessed 10 Apr 2020

3. World Health Organization (2020) Global surveillance for human infection with coronavirus disease (COVID-2019). Jan 31, 2020. World Health Organization, Geneva. Available via https://www. who.int/publications-detail/global-surveillance-for-humaninfection-with-novel-coronavirus-(2019-ncov). Accessed 10 Apr 2020

4. Spina S, Marrazzo F, Migliari M, Stucchi R, Sforza A, Fumagalli R (2020) The response of Milan's emergency medical system to the COVID-19 outbreak in Italy. Lancet 395:e49-e50

5. Stefanini GG, Azzolini E, Condorelli G (2020) Critical organizational issues for cardiologists in the COVID-19 outbreak: a frontline experience from Milan, Italy. Circulation 141:1597-1599

6. Remuzzi A, Remuzzi G (2020) COVID-19 and Italy: what next? Lancet 395:1225-1228
7. Grasselli G, Pesenti A, Cecconi M (2020) Critical care utilization for the COVID-19 outbreak in Lombardy, Italy: Early Experience and Forecast During an Emergency Response. JAMA. https://doi. org/10.1001/jama.2020.4031

8. Gazzetta Ufficiale della Repubblica Italiana (2020) DPCM 09 Marzo 2020 "Ulteriori disposizioni attuative del decreto-legge 23 febbraio 2020, n. 6, recante misure urgenti in materia di contenimento e gestione dell'emergenza epidemiologica da COVID-19, applicabili sull'intero territorio nazionale" (20A01605) (GU Serie Generale n.62 del 09-03-2020) DPCM 09.03.2020. Gazzetta Ufficiale della Repubblica Italiana, Rome. Available via https://www.gazzettaufficiale.it/atto/serie_generale/ caricaDettaglioAtto/originario?atto.dataPubblicazioneGazzetta $=$ 2020-03-09\&atto codiceRedazionale $=20$ A01558\&elenco 30 giorni=false. Accessed April 10, 2020

9. Gazzetta Ufficiale della Repubblica Italiana (2020) DPCM 22 Marzo 2020 "Ulteriori disposizioni attuative del decreto-legge 23 febbraio 2020 , n. 6 , recante misure urgenti in materia di contenimento e gestione dell'emergenza epidemiologica da COVID-19, applicabili sull'intero territorio nazionale" (20A01605) (GU Serie Generale n.76 del 22-03-2020) DPCM 22.03.2020. Gazzetta Ufficiale della Repubblica Italiana, Rome. Available via https://www.gazzettaufficiale.it/atto/serie_generale/ caricaDettaglioAtto/originario?atto.dataPubblicazioneGazzetta= 2020-03-22\&atto.codiceRedazionale $=20$ A01807\& elenco30 giorni=false. Accessed 10 Apr 2020

10. Signorelli C, Scognamiglio T, Odone A (2020) COVID-19 in Italy: impact of containment measures and prevalence estimates of infection in the general population. Acta Biomed 91:175-179

11. Zhou F, Yu T, Du R et al (2020) Clinical course and risk factors for mortality of adult inpatients with COVID-19 in Wuhan, China: a retrospective cohort study. Lancet 395:1054-1062

12. Carenzo L, Costantini E, Greco M et al (2020) Hospital surge capacity in a tertiary emergency referral centre during the COVID-19 outbreak in Italy. Anaesthesia. https://doi.org/10.1111/anae.15072

13. Boccia S, Ricciardi W, Ioannidis JPA (2020) What other countries can learn from Italy during the COVID-19 pandemic. JAMA Intern Med. https://doi.org/10.1001/jamainternmed.2020.1447

14. Charalel RA, McGinty G, Brant-Zawadzki M et al (2015) Interventional radiology delivers high-value health care and is an Imaging 3.0 vanguard. J Am Coll Radiol 12:501-506

15. Chandy PE, Nasir MU, Srinivasan S, Klass D, Nicolaou S, Babu SB (2020) Interventional radiology and COVID-19: evidencebased measures to limit transmission. Diagn Interv Radiol 26: 236-240

16. Zhu HD, Zeng CH, Lu J, Teng GJ (2020) COVID-19: what should interventional radiologists know and what can they do? J Vasc Interv Radiol. https://doi.org/10.1016/j.jvir.2020.03.022

17. Ierardi AM, Wood BJ, Gaudino C et al (2020) How to handle a COVID-19 patient in the angiographic suite. Cardiovasc Intervent Radiol 43:820-826

18. Hoe Gan W, Wah Lim J, Koh D (2020) Preventing intra-hospital infection and transmission of COVID-19 in healthcare workers. Saf Health Work. https://doi.org/10.1016/j.shaw.2020.03.001

19. Keeley AJ, Evans C, Colton $\mathrm{H}$ et al (2020) Roll-out of SARS-CoV2 testing for healthcare workers at a large NHS Foundation Trust in the United Kingdom, March 2020. Euro Surveill 25:2000433

20. Kandel N, Chungong S, Omaar A, Xing J (2020) Health security capacities in the context of COVID-19 outbreak: an analysis of International Health Regulations annual report data from 182 countries. Lancet 395:1047-1053

21. Gogna A, Punamiya S, Gopinathan A et al (2020) Preparing IR for COVID-19: the Singapore experience. J Vasc Interv Radiol. https:// doi.org/10.1016/j.jvir.2020.03.021

22. Society of Interventional Radiology (2020) COVID-19 case classification. Society of Interventional Radiology, Fairfax (VA). 
Available via https://www.sirweb.org/practice-resources/toolkits/ covid-19-toolkit/covid-19-case-classification. Accessed 10 Apr 2020

23. Da Zhuang K, Tan BS, Tan BH, Too CW, Tay KH (2020) Old threat, new enemy: is your interventional radiology service ready for the coronavirus disease 2019? Cardiovasc Intervent Radiol 43: 665-666

24. Pedicelli A, Valente I, Pilato F, Distefano M, Colosimo C (2020) Stroke priorities during COVID-19 outbreak: acting both fast and safe. J Stroke Cerebrovasc Dis. https://doi.org/10.1016/j. jstrokecerebrovasdis.2020.104922

25. Ma QX, Shan H, Zhang HL, Li GM, Yang RM, Chen JM (2020) Potential utilities of mask-wearing and instant hand hygiene for fighting SARS-CoV-2. J Med Virol. https://doi.org/10.1002/jmv. 25805
26. Nicola M, O'Neill N, Sohrabi C, Khan M, Agha M, Agha R (2020) Evidence based management guideline for the COVID-19 pandemic - review article. Int J Surg 77:206-216

27. World Health Organization (2020) Rational use of personal protective equipment for coronavirus disease (COVID - 19) World Health Organization - Interim Guidance - 27 February 2020. World Health Organization, Geneva. Available via https://apps.who.int/ iris/bitstream/handle/10665/331215/WHO-2019-nCov-IPCPPE_ use-2020.1-eng.pdf. Accessed 10 Apr 2020

28. Roda WC, Varughese MB, Han D, Li MY (2020) Why is it difficult to accurately predict the COVID-19 epidemic? Infect Dis Model 5: 271-281

Publisher's note Springer Nature remains neutral with regard to jurisdictional claims in published maps and institutional affiliations. 